hep-ph/9512278

MIT-CTP-2478

\title{
Phenomenological Constraints on the Higgs as Pseudo-Goldstone Boson Mechanism in Supersymmetric GUT Theories
}

\author{
Csaba Csáki and Lisa Randall[ \\ Center for Theoretical Physics \\ Laboratory for Nuclear Science and Department of Physics \\ Massachusetts Institute of Technology \\ Cambridge, MA 02139, USA
}

\begin{abstract}
There are few robust solutions to the doublet-triplet splitting problem in supersymmetric GUT theories. One of the more promising solutions is the Higgs as pseudo-Goldstone boson mechanism. In its minimal implementation, such a solution places an additional restriction on the parameter space of the minimal supersymmetric standard model. A testable consequence of this constraint is an equation for $\tan \beta$. We present this restriction and study its solutions in order to constrain the allowed parameter space. Thus the assumptions on the GUT scale Higgs sector should yield testable predictions for weak scale physics. If the SUSY parameters are measured then it should be possible to check the predictions, yielding insight into GUT scale physics.
\end{abstract}

\footnotetext{
${ }^{1}$ Supported in part by DOE under cooperative agreement \#DE-FC02-94ER40818.

${ }^{2}$ NSF Young Investigator Award, Alfred P. Sloan Foundation Fellowship, DOE Outstanding Junior Investigator Award.
} 


\section{Introduction}

The unification of couplings in the MSSM is strong evidence for supersymmetric grand unified theories (SUSY GUT's). However a credible SUSY GUT should incorporate a solution to the doublet-triplet splitting problem and the associated $\mu$-problem.

The Higgs as pseudo-Goldstone boson (PGB) scheme elegantly solves both problems [1]. In this solution the Higgs fields are light (of the order of the weak scale) because an additional global symmetry is spontaneously broken implying the presence of some massless Goldstone bosons. By suitable choice of the gauge and global symmetry groups one can achieve a situation in which the pseudo-Goldstone bosons are exactly one pair of $\mathrm{SU}(2)$ doublets to be identified with the Higgs fields of the MSSM [1-9]. At the same time this mechanism naturally generates a $\mu$-term.

In this paper we investigate the restrictions that the assumption that the $\mu$-term is generated purely by the Higgs as PGB mechanism places on the low energy physics. Because of an additional constraint on the Higgs sector $\tan \beta$ is not an independent parameter. We impose this constraint and check whether a suitable standard model minimum exists. This will result in an equation for $\tan \beta$ which is given in eq. 3.15.

We solve this equation numerically and give the resulting range of $\tan \beta$. The parameter range where this equation can be satisfied is also displayed.

Thus we will show how our assumptions on the GUT physics together with our present knowledge of weak scale phenomenology constrain the parameters of weak scale supersymmetry. This way we could get information on the GUT scale physics if the MSSM parameters are ultimately measured in future colliders.

The paper is organized as follows: in Section 2 we review how a $\mu$-term is generated in the Higgs as PGB scheme. Section 3 contains the analysis of the extra constraint - which reduces the number of independent MSSM parameters by one. In Section 4 we discuss our numerical results and the resulting allowed parameter space of the MSSM. The consequences of these results for realistic SUSY GUT models is discussed in Section 5. We conclude in Section 6 .

\section{The $\mu$-term from the Higgs as PGB scheme}

In the Higgs as PGB solution to the doublet-triplet splitting problem one assumes that there is an additional global symmetry which, after spontaneous breaking, ensures the lightness of the Higgs particles [1]. For example, in the originally proposed model the $\mathrm{SU}(5)$ gauge group is enlarged to an $\mathrm{SU}(6)$ global symmetry containing the gauged $\mathrm{SU}(5)$. While the $\mathrm{SU}(6)$ breaks to $\mathrm{SU}(4) \otimes \mathrm{SU}(2) \otimes \mathrm{U}(1)$, the gauged $\mathrm{SU}(5)$ breaks to the SM group $\mathrm{SU}(3) \otimes \mathrm{SU}(2) \otimes \mathrm{U}(1)$, leaving one pair of $\mathrm{SU}(2)$ doublets as uneaten Goldstone bosons [1, 2].

However only one of the scalars in the chiral superfields is a genuine Goldstone boson; 
the other scalar is massless only on account of supersymmetry. Thus it is not surprising that the soft breaking terms (which do preserve the extra global symmetry) generate mass terms for these non-Goldstone boson light fields and a supersymmetric $\mu$-term for the Higgs fields.

In this section we will explicitly demonstrate how this mechanism works in the above mentioned $\mathrm{SU}(5)$ model with $\mathrm{SU}(6)$ global symmetry. Although this model is not the most aesthetic in that it requires tuning the $\mathrm{SU}(5)$ couplings, the simplicity of this model makes it a good example to illustrate the generic features of the Higgs as PGB mechanism. We will also see in section 5 that this model can be an effective theory for some energy range for the more realistic model of refs. [4, 6, 7, 8, 9]. All the results in this section apply more generally as has been shown in ref. [1].

The Higgs sector of this model consists of one adjoint of SU(6), denoted by $\Phi$,

$$
\Phi=24+5+\overline{5}+1=\Sigma+H+\bar{H}+S
$$

under $\mathrm{SU}(5)$. The explicit realization of this decomposition is given in the following way:

$$
\Phi=\left(\begin{array}{cc}
-\frac{5}{\sqrt{30}} S & H \\
\bar{H} & \Sigma+\frac{1}{\sqrt{30}} S
\end{array}\right) .
$$

The superpotential is given by

$$
W(\Phi)=\frac{1}{2} M \operatorname{Tr} \Phi^{2}+\frac{1}{3} \lambda \operatorname{Tr} \Phi^{3} .
$$

The VEV is given by

$$
\langle\Phi\rangle=\frac{M}{\lambda}\left(\begin{array}{cccccc}
1 & & & & & \\
& 1 & & & & \\
& & 1 & & & \\
& & & 1 & & \\
& & & & -2 & \\
& & & & & -2
\end{array}\right),
$$

which breaks the gauged $\mathrm{SU}(5)$ to $\mathrm{SU}(3) \otimes \mathrm{SU}(2) \otimes \mathrm{U}(1)$, and leaves one pair of Goldstone bosons uneaten, which can be identified as the $\mathrm{SU}(2)$ doublets in $H$ and $\bar{H}$. The scalar potential after the inclusion of the soft breaking terms is given by

$$
V(\Phi)=\operatorname{Tr}\left|M \Phi+\lambda\left(\Phi^{2}-\frac{1}{6} \operatorname{Tr} \Phi^{2}\right)\right|^{2}+\left(A_{\Phi} \lambda \frac{1}{3} \operatorname{Tr} \Phi^{3}+B_{\Phi} M \frac{1}{2} \operatorname{Tr} \Phi^{2}+\text { h.c. }\right)+m_{0}^{2} \operatorname{Tr}|\Phi|^{2} .
$$

Now the $\Phi \mathrm{VEV}$ is shifted to

$$
\langle\Phi\rangle=\frac{1}{\lambda}\left[M+\left(A_{\Phi}-B_{\Phi}\right)+\frac{1}{M}\left(3 A_{\Phi} B_{\Phi}-A_{\Phi}^{2}-2 B_{\Phi}^{2}-m_{0}^{2}\right)+\mathcal{O}\left(\frac{1}{M^{2}}\right)\right] .
$$


After substituting $\langle\Phi\rangle$ into the potential one finds a mass term for the doublets in $H, \bar{H}$ (denoted by $h_{u}, h_{d}$ )

$$
\left|\frac{h_{u}-h_{d}^{\dagger}}{\sqrt{2}}\right|^{2}\left[2 m_{0}^{2}+2\left(A_{\Phi}-B_{\Phi}\right)^{2}\right]
$$

while at the same time the shift in the $\Phi \mathrm{VEV}$ generates a mass term for the higgsinos $\tilde{h_{u}}$ and $\tilde{h_{d}}$ of the form

$$
\left(A_{\Phi}-B_{\Phi}\right) \tilde{h_{u}}{ }^{c} \tilde{h_{d}}+h . c . .
$$

Thus, as it was shown in general in ref. [1], there is a supersymmetric $\mu$-term generated by this mechanism, which is usually a model dependent function of the soft breaking parameters (in the above described model $\mu=A_{\Phi}-B_{\Phi}$ ). The other important lesson from this example is that the combination $1 / \sqrt{2}\left(h_{u}+h_{d}^{\dagger}\right)$ is the genuine Goldstone boson with no mass term at the GUT scale even after inclusion of the soft breaking terms [1]. Such a mass term is generated only by the explicit symmetry breaking loop corrections.

Thus, the general conclusion is that the Higgs as PGB mechanism generates the following mass term for the MSSM Higgs fields at the GUT scale:

$$
\left.V\left(h_{u}, h_{d}\right)\right|_{M_{G U T}}=\left(m_{0}^{2}+\mu^{2}\right)\left|h_{u}-h_{d}^{\dagger}\right|^{2}+\text { D-terms, }
$$

where $m_{0}$ is the soft breaking mass parameter introduced in eq. 2.5, while $\mu$ is a function of all soft breaking parameters.

The general Higgs potential in the MSSM is given by

$$
\left.V\left(h_{u}, h_{d}\right)\right|_{\Lambda}=m_{1}^{2}(\Lambda) h_{d}^{\dagger} h_{d}+m_{2}^{2}(\Lambda) h_{u}^{\dagger} h_{u}+m_{3}^{2}(\Lambda)\left(h_{u} h_{d}+h . c .\right)+\text { D-terms }
$$

where we have explicitly displayed the scale $(\Lambda)$ dependence of the parameters and where

$$
\begin{aligned}
& m_{1}^{2}(\Lambda)=m_{d}^{2}(\Lambda)+\mu^{2}(\Lambda), \\
& m_{2}^{2}(\Lambda)=m_{u}^{2}(\Lambda)+\mu^{2}(\Lambda), \\
& m_{3}^{2}(\Lambda)=B(\Lambda) \mu(\Lambda),
\end{aligned}
$$

$m_{u}^{2}, m_{d}^{2}$ are the running soft breaking mass terms for the up- and down-type Higgses, $\mu(\Lambda)$ is the running $\mu$-parameter, while $B(\Lambda)$ is the running soft breaking parameter corresponding to the $\mu$-term. From eq. 2.9 one can see that the specific $\mu$-term generated by the Higgs as PGB mechanism requires that the boundary condition

$$
m_{d}^{2}\left(M_{G}\right)+\mu^{2}\left(M_{G}\right)=m_{u}^{2}\left(M_{G}\right)+\mu^{2}\left(M_{G}\right)=-B\left(M_{G}\right) \mu\left(M_{G}\right)
$$

is satisfied. If one assumes universal soft breaking terms at the GUT-scale, then the first of the equations is automatically satisfied, and we have one additional constraint equation. 
As noted already in refs. [1, 2, 5, 6], eq. 2.12 means that the number of free parameters in the MSSM is reduced by one. However we have not seen in any of the previous analyses an explicit determination of the results of this additional restriction.

Already the authors of the first papers on this subject noted the importance of eq. 2.12, and analyzed its consequences. However in these papers [2, 5] a very specific form of the soft breaking terms was assumed and therefore the consequences were not sufficiently general. The authors of ref. [6] also provide an analysis of the constraint of eq. 2.12. However their method is not described in sufficient detail for us to compare the results.

\section{Analysis of the constraint arising from the Higgs as PGB mechanism}

As we saw in the previous section, the specific form of the $\mu$-term generated by the Higgs as PGB mechanism implies the following constraint on the running mass parameters:

$$
m_{d}^{2}(M)+\mu^{2}(M)=m_{u}^{2}(M)+\mu^{2}(M)=-B(M) \mu(M)
$$

where $M$ is the scale where the additional global symmetry which gives rise to the light Higgses is broken. We make the following assumptions:

A. The $\mu$-term is generated purely by the Higgs as PGB mechanism implying the constraint 3.1.

B. The form of the constraint 3.1 remains valid at the GUT-scale.1

Usually the number of free parameters in the MSSM (assuming universal soft breaking terms at the GUT scale and gaugino unification) is $5+1$, where the $5+1$ are:

1. $m_{0}$ - the universal soft breaking mass term,

2. $A_{0}$ - the trilinear soft breaking term,

3. $M_{1 / 2}$ - the gaugino mass,

4. $\lambda_{t}$ - the top Yukawa coupling,

5. $\tan \beta$ - the ratio of Higgs VEV's,

and the extra parameter is the sign of the $\mu$ parameter.

We can see that this set does not contain either $\mu$ or $B$, since they are determined (at the weak scale) from the requirement of electroweak symmetry breaking (see e.g. [11]):

$$
\begin{aligned}
& \mu^{2}\left(M_{Z}\right)=\frac{\bar{m}_{d}^{2}-\bar{m}_{u}^{2} \tan ^{2} \beta}{\tan ^{2} \beta-1}-\frac{1}{2} M_{Z}^{2}, \\
& B\left(M_{Z}\right) \mu\left(M_{Z}\right)=\frac{1}{2} \sin 2 \beta\left(\bar{m}_{1}^{2}+\bar{m}_{2}^{2}\right),
\end{aligned}
$$

\footnotetext{
${ }^{1}$ Our results do not change significantly when allowing running between $M_{P}$ and $M_{G}$. See the end of Section 5.
} 
where $\bar{m}_{d}^{2}, \bar{m}_{u}^{2}, \bar{m}_{1}^{2}, \bar{m}_{2}^{2}$ are the 1-loop corrected values of the above defined soft breaking mass parameters evaluated at the weak scale.

This means that the constraint eq. 3.1 will determine one additional parameter of the 5 , but in a nontrivial way. In our analysis we choose $m_{0}, A_{0}, M_{1 / 2}, \lambda_{t}$ and the sign of $\mu$ to be the independent parameters and $\tan \beta$ will be evaluated in the following way: given the 4 input parameters, one can calculate the soft breaking mass terms at the weak scale through RG running. Thus, we will have an expression for $\mu^{2}\left(M_{Z}\right)$ and $B\left(M_{Z}\right)$ as a function of $\tan \beta$ for every set of input parameters. Then we scale these expressions back to the GUT-scale and require that eq. 3.1 is satisfied. This will yield an equation for $\tan \beta$. In our analysis we use the one loop RGE's for the MSSM, retaining only the top Yukawa coupling and the gauge couplings. In this case the RGE's can be solved analytically with the exception of one function, where numerical integration is necessary. The approximate analytical expressions are [10, 12]:

$$
\begin{aligned}
& m_{u}^{2}=m_{0}^{2}+0.52 M_{1 / 2}^{2}+\Delta m^{2} \\
& m_{d}^{2}=m_{0}^{2}+0.52 M_{1 / 2}^{2} \\
& \mu^{2}\left(M_{Z}\right)=2 \mu_{0}^{2}\left(1-\frac{Y_{t}}{Y_{f}}\right)^{1 / 2} \\
& B\left(M_{Z}\right)=B_{0}-\frac{A_{0}}{2} \frac{Y_{t}}{Y_{f}}+M_{1 / 2}\left(1.2 \frac{Y_{t}}{Y_{f}}-0.6\right),
\end{aligned}
$$

where

$$
\begin{gathered}
\Delta m^{2}=-\frac{3}{2} m_{0}^{2} \frac{Y_{t}}{Y_{f}}+2.3 A_{0} M_{1 / 2} \frac{Y_{t}}{Y_{f}}\left(1-\frac{Y_{t}}{Y_{f}}\right)-\frac{A_{0}^{2}}{2} \frac{Y_{t}}{Y_{f}}\left(1-\frac{Y_{t}}{Y_{f}}\right)+M_{1 / 2}^{2}\left[-7 \frac{Y_{t}}{Y_{f}}+3\left(\frac{Y_{t}}{Y_{f}}\right)^{2}\right]_{(3.4)}, \\
Y_{t}=\lambda_{t}^{2} / 4 \pi \\
Y_{t}=\frac{2 \pi Y_{t}\left(M_{G}\right) E(t)}{2 \pi+3 Y_{t}\left(M_{G}\right) F(t)}, \\
E(t)=\left(1+\beta_{3} t\right)^{\frac{16}{3 b_{3}}}\left(1+\beta_{2} t\right)^{\frac{3}{b_{2}}}\left(1+\beta_{1} t\right)^{\frac{13}{9 b_{1}}}, \\
\beta_{i}=\alpha_{i}\left(M_{G}\right) b_{i} / 4 \pi, \\
t=\log \left(M_{G} / \Lambda\right)^{2}, \\
F(t)=\int_{0}^{t} E\left(t^{\prime}\right) d t^{\prime}, \\
Y_{f}=\frac{2 \pi E(t)}{3 F(t)},
\end{gathered}
$$


for $t=\log \left(M_{G} / M_{Z}\right)^{2}, M_{G}=2 \cdot 10^{16}$ one has $E \simeq 14, F \simeq 293$ in the MSSM. Putting these together with the constraint eq. 3.1 and with eq. 3.2 for electroweak breaking one gets the following equation for $\tan \beta$ :

$$
\begin{aligned}
& m_{0}^{2}+\frac{1}{2\left(1-\frac{Y_{t}}{Y_{f}}\right)^{1 / 2}}\left(-m_{0}^{2}-0.52 M_{1 / 2}^{2}-\Delta m^{2} \frac{\tan ^{2} \beta}{\tan ^{2} \beta-1}-\frac{M_{Z}^{2}}{2}\right)= \\
& -\frac{1}{\sqrt{2}\left(1-\frac{Y_{t}}{Y_{f}}\right)^{1 / 4}}\left\{-\frac{\tan \beta}{1+\tan ^{2} \beta}\left(\Delta m^{2} \frac{\tan ^{2} \beta+1}{\tan ^{2} \beta-1}+M_{Z}^{2}\right)\right. \\
& \pm\left[\frac{A_{0}}{2} \frac{Y_{t}}{Y_{f}}-M_{1 / 2}\left(1.2 \frac{Y_{t}}{Y_{f}}-0.6\right)\right]\left(-m_{0}^{2}-0.52 M_{1 / 2}^{2}\right. \\
& \left.\left.-\Delta m^{2} \frac{\tan ^{2} \beta}{\tan ^{2} \beta-1}-\frac{M_{Z}^{2}}{2}\right)^{1 / 2}\right\} .
\end{aligned}
$$

When solving this equation one has to be careful about the sign of $\tan \beta$. In the MSSM one can fix $\tan \beta$ to be positive, because for negative $\tan \beta$ one can redefine the phase of one of the Higgs fields to absorb a minus sign and thus changing the sign of the $B \mu$ term in eq. 2.10. However in our case the constraint of eq. 2.12 is not invariant under this phase redefinition. As a result eq. 3.12 is not invariant under $\tan \beta \rightarrow-\tan \beta$, thus as opposed to the MSSM one loses generality by restricting to positive $\tan \beta$. Therefore we solve eq. 3.12 separately for positive and negative $\tan \beta$.

The \pm in eq. 3.12 stands for the two possible signs of the $\mu$ parameter. Note however, that eq. 3.12 does not yet include the corrections to $m_{u}^{2}$ and $m_{d}^{2}$ arising from the one loop corrections to the effective potential which are known to be significant and which should be incorporated. The expressions for these corrections are:

$$
\begin{aligned}
& \Delta m_{u}^{2}=\frac{\partial \Delta V}{\partial v_{u}^{2}}, \\
& \Delta m_{d}^{2}=\frac{\partial \Delta V}{\partial v_{d}^{2}},
\end{aligned}
$$

and we retain only the top-stop loops for $\Delta V$ :

$$
\Delta V=\frac{3}{16 \pi^{2}}\left[\frac{1}{2} m_{\tilde{t}_{1}}^{4}\left(\log \left(\frac{m_{\tilde{t}_{1}}^{2}}{\Lambda^{2}}\right)-\frac{3}{2}\right)+\frac{1}{2} m_{\tilde{t}_{2}}^{4}\left(\log \left(\frac{m_{\tilde{t}_{2}}^{2}}{\Lambda^{2}}\right)-\frac{3}{2}\right)-m_{t}^{4}\left(\log \left(\frac{m_{t}^{2}}{\Lambda^{2}}\right)-\frac{3}{2}\right)\right]
$$

where $m_{\tilde{t}_{1,2}}$ are the stop masses and $m_{t}$ is the top mass. Eq. 3.12 is then modified to be

$$
m_{0}^{2}+\frac{1}{2\left(1-\frac{Y_{t}}{Y_{f}}\right)^{1 / 2}}\left(-m_{0}^{2}-0.52 M_{1 / 2}^{2}-\Delta m^{2} \frac{\tan ^{2} \beta}{\tan ^{2} \beta-1}-\frac{M_{Z}^{2}}{2}+\frac{\Delta m_{d}^{2}}{\tan ^{2} \beta-1}\right.
$$




$$
\begin{aligned}
& \left.-\frac{\Delta m_{u}^{2} \tan ^{2} \beta}{\tan ^{2} \beta-1}\right)=-\frac{1}{\sqrt{2}\left(1-\frac{Y_{t}}{Y_{f}}\right)^{1 / 4}}\left\{-\frac{\tan \beta}{1+\tan ^{2} \beta}\left[\left(\Delta m^{2}+\Delta m_{u}^{2}-\Delta m_{d}^{2}\right) \frac{\tan ^{2} \beta+1}{\tan ^{2} \beta-1}\right.\right. \\
& \left.+M_{Z}^{2}\right] \pm\left[\frac{A_{0}}{2} \frac{Y_{t}}{Y_{f}}-M_{1 / 2}\left(1.2 \frac{Y_{t}}{Y_{f}}-0.6\right)\right]\left(-m_{0}^{2}-0.52 M_{1 / 2}^{2}-\Delta m^{2} \frac{\tan ^{2} \beta}{\tan ^{2} \beta-1}-\frac{M_{Z}^{2}}{2}+\right. \\
& \left.\left.\frac{\Delta m_{d}^{2}}{\tan ^{2} \beta-1}-\frac{\Delta m_{u}^{2} \tan ^{2} \beta}{\tan ^{2} \beta-1}\right)^{1 / 2}\right\} .
\end{aligned}
$$

\section{Results}

We have shown in the previous section that the fact that the $\mu$-term is generated by the Higgs as PGB mechanism implies eq. 3.12 for $\tan \beta$.

We note that eq. 3.12 is invariant under the transformation $\mu \rightarrow-\mu, M_{1 / 2} \rightarrow-M_{1 / 2}$ and $A_{0} \rightarrow-A_{0}$. Therefore one can fix $\mu<0$, and then the solutions corresponding to positive $\mu$ are obtained by taking $M_{1 / 2} \rightarrow-M_{1 / 2}$ and $A_{0} \rightarrow-A_{0}$. Consequently we will show four plots; the first two corresponding to $\tan \beta>0$ and both signs for $M_{1 / 2}$ (Figs. 1.c and 1.d). Subsequently we will give the two plots corresponding to $\tan \beta<0$, with $M_{1 / 2}>0$ in Fig. 2 and $M_{1 / 2}<0$ in Fig. 3. In all these plots we will have $\mu<0$ fixed. All $\mu>0$ solutions can be obtained as described above.

The values of $m_{0}$ and $\left|M_{1 / 2}\right|$ are bounded from below in order to ensure that the sparticle masses obey the experimental limits [14]. Thus one has to combine the experimental lower bounds on the sparticle masses and the requirement that there is a solution to eq. 3.15 to get the possible parameter range of the MSSM.

We solved equation 3.15 numerically for the small $\tan \beta$ regime (we take $1<\tan \beta<15$; large $\tan \beta$ would require a fine tuning of order $1 / \tan \beta$ in the Higgs sector [13] contrary to the spirit of not tuning parameters). In the following we summarize the main features of the solutions.

If one fixes $\tan \beta>0$ then the only viable solutions fulfilling the experimental constraints correspond to

-large values of $\left|A_{0}\right|$

-relatively small values of $m_{0}$ and $\left|M_{1 / 2}\right|$.

This is illustrated in Fig. 1 where we display the allowed parameter space for a fixed value of $\lambda_{t}$ and different fixed values of $m_{0}$. One gets the same type of plots for other fixed values of $\lambda_{t}$. Figs. 1. a and $\mathrm{b}$ are presented to ease the reading of the subsequent plots. They both display the $m_{0}=60$ case with detailed explanation of the allowed parameter space. Figs. 1. c and $d$ are the same but for four different values of $m_{0}$.

However there is an important potential problem with these solutions: the existence of charge and/or color breaking (CCB) minima of the potential of the sleptons and squarks. We use the "traditional" condition for the absence of such minima 15 but we evaluate this 
condition not at the GUT scale but at the weak scale. This yields the following conditions for the weak scale parameters:

$$
\begin{aligned}
& A_{e}^{2}<3\left(m_{\tilde{L}}^{2}+m_{\tilde{e}}^{2}+m_{d}^{2}\right) \\
& A_{d}^{2}<3\left(m_{\tilde{Q}}^{2}+m_{\tilde{d}}^{2}+m_{d}^{2}\right) \\
& A_{u}^{2}<3\left(m_{\tilde{Q}}^{2}+m_{\tilde{u}}^{2}+m_{u}^{2}\right),
\end{aligned}
$$

where the $A_{e}, A_{d}$ and $A_{u}$ refer to the soft breaking trilinear terms of a given interaction (that is $A_{0}$ scaled down to the weak scale) and the m's refer to the soft breaking scalar mass terms also evaluated at the weak scale. We have calculated these parameters in the same one loop approximation (that is including only loops with gauge or top Yukawa couplings), and plotted the allowed region (that is the region where the inequalities 4.1 are satisfied). It is well known that these conditions are neither sufficient nor necessary to avoid the presence of CCB vacua 15. The full analysis for the absence of these particular CCB vacua has been recently done in [16. We have checked that these results are sufficently close to those of the full analysis given in ref. [16], with the results of ref. [16] being always even more restrictive than those based on our analysis.

Furthermore even if a CCB vacuum exists that is a global minimum of the scalar potential one has to calculate the tunneling rate from the false MSSM vacuum to the real CCB vacuum for each such solution and only if that is large can one exclude a given point in the parameter space. Thus it is clear that eq. 4.1 is not the full story. However it can be used as an approximate indicator for the presence of CCB vacua. If one is very far outside the allowed region allowed by 4.1 then that point on the parameter space can be safely excluded. If one is deep inside the allowed region CCB vacua are probably not dangerous.

In Fig. 1 we also display the CCB bounds obtained from eqs. 4.1, along with the allowed parameter space. As one can see from Fig. 1, all these solutions to equation 3.15 lay outside the bounds of 4.1 and thus CCB poses a threat to the entire allowed parameter space. Therefore if we take these CCB bounds seriously we have to discard these solutions. This conclusion is not altered if we take different values of $\lambda_{t}$.

In this case we can conclude that one needs to take $\tan \beta<0$. If we again fix $\mu<0$ as before. We will get two type of solutions for this case. The solutions to the $M_{1 / 2}>0$ case resemble very much the solutions of the previous case; that is one needs to have large $\left|A_{0}\right|$ and small $m_{0}$ and $M_{1 / 2}$. However now larger values of $M_{1 / 2}$ and $m_{0}$ are possible, but the overlap with the CCB allowed region is still very small as illustrated in Fig. 2. (There is still no overlap for small values of $m_{0}$ 's and tiny overlap for large values.)

The final possibility (negative $\tan \beta$, negative $\mu$ and negative $M_{1 / 2}$ ) is not so restrictive. In this case one does not get an upper bound on $\left|M_{1 / 2}\right|$ and $m_{0}$; instead one gets a lower bound on $\left|M_{1 / 2}\right|$ (which is however more constraining then the usual experimental bounds in the MSSM). One gets a large overlap with the CCB allowed region. This region of overlap 


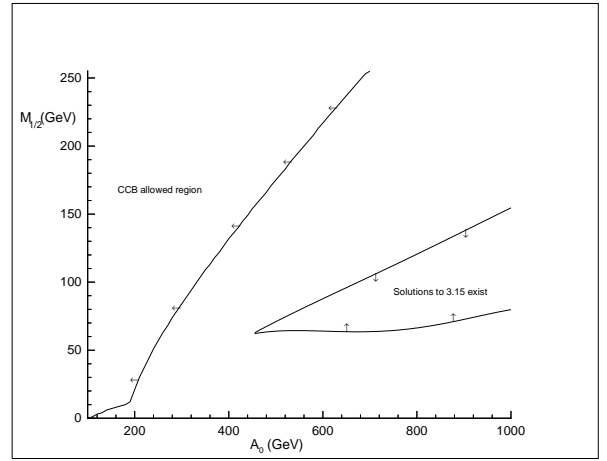

Fig. 1.a

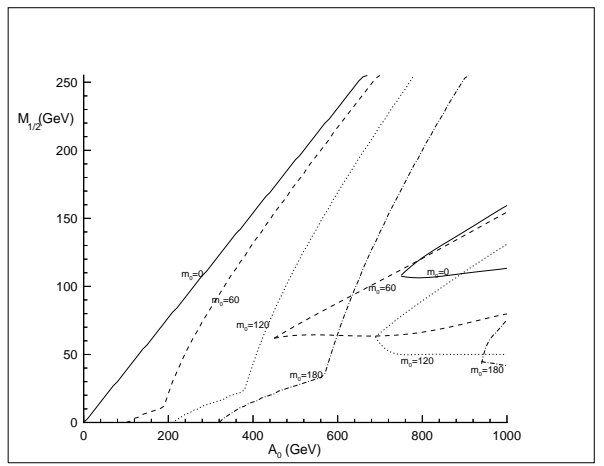

Fig. 1.c

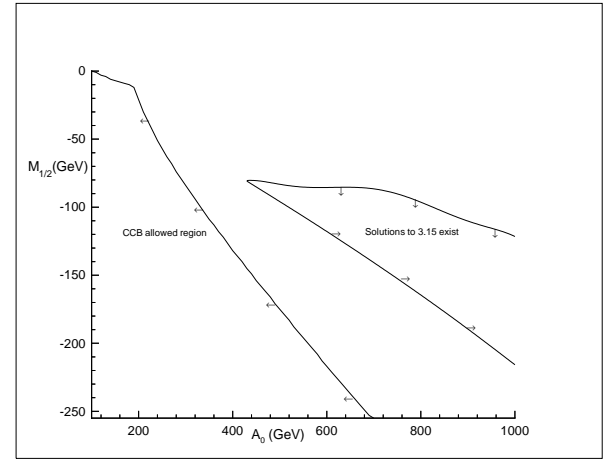

Fig. 1.b

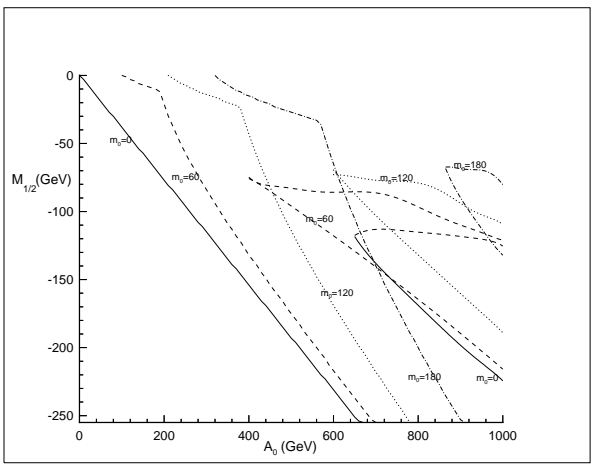

Fig. 1.d

Figure 1: The allowed MSSM parameter space for a fixed value of $\lambda_{t}\left(\lambda_{t}=1.2\right)$ and for different fixed values of $m_{0}$ with $\tan \beta>0$.

a: $m_{0}=60 \mathrm{GeV}, M_{1 / 2}>0$. The curve on the right of the plot gives the region where equation 3.15 can be satisfied with the above mentioned parameters, while the curve on the left gives the region where CCB vacua are absent.

b: the same as in 1.a for $M_{1 / 2}<0$. In both cases $\mu<0$. As explained in the text the $\mu>0$ solutions can be obtained by taking $M_{1 / 2} \rightarrow-M_{1 / 2}$ and $A_{0} \rightarrow-A_{0}$ simultaneously.

c: As in 1.a for varying $m_{0}$. In both Figs. 1.c and 1.d the solid line corresponds to $m_{0}=0 \mathrm{GeV}$, the dashed to $m_{0}=60 \mathrm{GeV}$, the dotted to $m_{0}=120 \mathrm{GeV}$ and the dash-dotted to $m_{0}=180 \mathrm{GeV}$.

d: As in 1.b for varying $m_{0}$. 


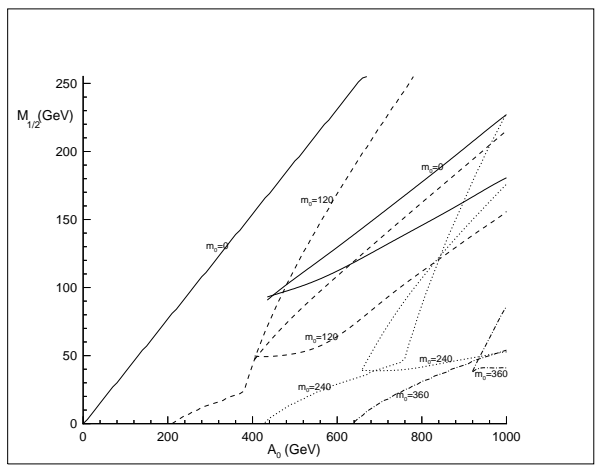

Figure 2: The same as Fig. 1 but for $\tan \beta<0$ and positive $M_{1 / 2}$. The solid line corresponds to $m_{0}=0 \mathrm{GeV}$, the dashed to $m_{0}=120 \mathrm{GeV}$, dotted to $m_{0}=240 \mathrm{GeV}$ and the dash-dotted to $m_{0}=360 \mathrm{GeV}$. One can see that the overlap of the solutions with the CCB allowed regions is very tiny.

is however much more restricted than the region of parameters allowed in the MSSM. This is illustrated in Fig. 3 for different values of $m_{0}$ and fixed $\lambda_{t}$. Figs. 3.a and 3.c are again presented to ease the reading of the other two plots, with detailed explanation of the allowed region. Note in Figs. 3.b and 3.d that for $m_{0}=500,1000 \mathrm{GeV}$ one does not get any restriction from the $\mathrm{CCB}$ bounds if $\left|A_{0}\right|<1000 \mathrm{GeV}$. Also note that for large values of $m_{0}$ the MSSM bound on $M_{1 / 2}$ is independent of $m_{0}$. Different values of $\lambda_{t}$ yield similar plots, with the curves somewhat shifted to the right (towards larger values of $A_{0}$ ). This is illustrated in Figs. 3.b and 3.d.

In summary we find that most of the possible solutions to eq. 3.15 obeying the CCB bounds correspond to the $\tan \beta, M_{1 / 2}, \mu<0$ case with the allowed parameter space displayed in Fig. 3.

Since $\tan \beta$ is not a free parameter of the theory it is not surprising that the range of values $\tan \beta$ can take on is much smaller than in the MSSM. There any low value of $\tan \beta$ not too close to 1 can be acceptable for fixed $A_{0}$ and $m_{0}$ if one varies $M_{1 / 2}$. In our case however $\tan \beta$ is the solution to eq. 3.15 and thus will in general not take all values. This is illustrated in Fig. 4, where we display the allowed range of $\tan \beta$ for fixed values of $\lambda_{t}$ and $m_{0}$, while $\left|M_{1 / 2}\right|$ is allowed to vary in the range of $0,800 \mathrm{GeV}$. One can see that one finds a smaller region of acceptable vacua than the MSSM together with the experimental constraints would allow.

To conclude this section we summarize the consequences of our analysis. We have seen that the boundary condition 2.12 together with the requirement of an acceptable SM minimum will determine $\tan \beta$ from other input parameters. We have seen that this equation for $\tan \beta$ does not always have solutions which excludes some regions of the MSSM parameter 


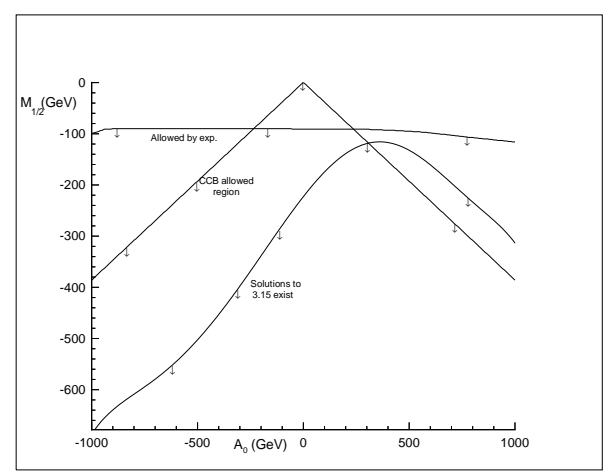

Fig. 3.a

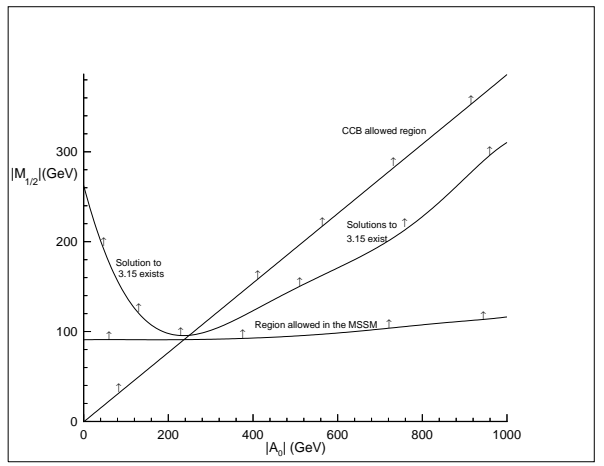

Fig. 3.c

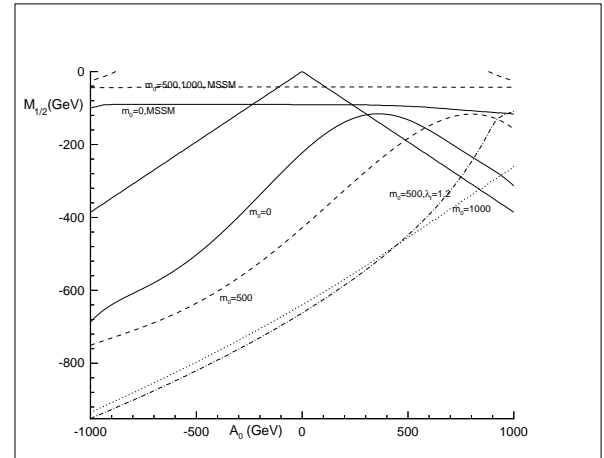

Fig. 3.b

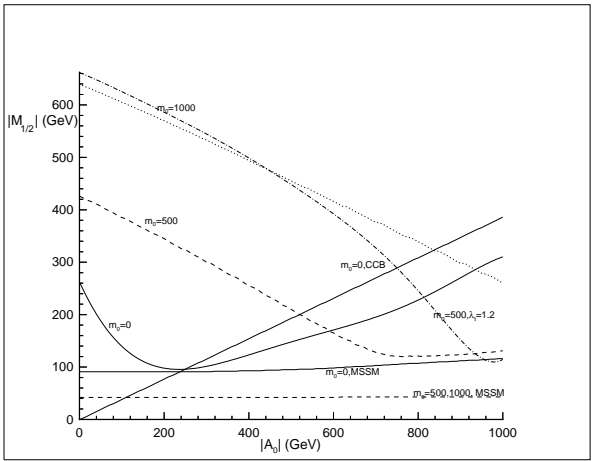

Fig. 3.d

Figure 3: The allowed region of parameters for $\tan \beta<0$ and negative $M_{1 / 2} \cdot \lambda_{t}=0.8$ for all four plots (except in Figs. 3.b and 3.d where explicitly stated) a: $m_{0}=0 \mathrm{GeV}$. The upper straight line corresponds to the bound on $M_{1 / 2}$ in the MSSM, if the values of $\tan \beta$ are varied between 1 and 15 . The $\Lambda$-shaped curve on the top of the plot gives the region allowed by the absence of CCB vacua, while the lower line corresponds to the bound on $M_{1 / 2}$ obtained from equation 3.15. b: The same as in Fig. 3.a but for different values of $m_{0}$. The solid lines corresponds to $m_{0}=0 \mathrm{GeV}$, the dashed lines to $m_{0}=500 \mathrm{GeV}$ and the dotted line to $m_{0}=1000 \mathrm{GeV}$. Note that one does not get any restriction from the CCB bounds for $m_{0}=500,1000 \mathrm{GeV}$ when $\left|A_{0}\right|<1000$ $\mathrm{GeV}$. Also note that for large values of $m_{0}$ the MSSM bound on $M_{1 / 2}$ is independent of $m_{0}$. The dash-dotted line corresponds to $m_{0}=500 \mathrm{GeV}$, but with $\lambda_{t}=1.2$. The increase in $\lambda_{t}$ results in the shift of the curves towards larges values of $A_{0}$. c: The bounds on the absolute value of $M_{1 / 2}$ as a function of the absolute value of $A_{0}$ obtained from Figs. 3.a-b. 3.c gives the $m_{0}=0$ case, where the lower straight curve is the MSSM bound, the upper straight curve is the bound from CCB, while the third curve in the middle is the bound obtained by requiring that 3.15 has a viable solution. d: The same as in c, where $m_{0}=0 \mathrm{GeV}$ corresponds to the solid lines, $m_{0}=500 \mathrm{GeV}$ to the dashed lines and $m_{0}=1000 \mathrm{GeV}$ to the dotted line. The dash-dotted line corresponds to $m_{0}=500 \mathrm{GeV}$ but $\lambda_{t}=1.2$. 


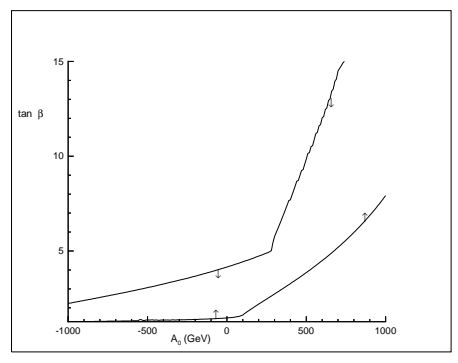

Fig. 4.a

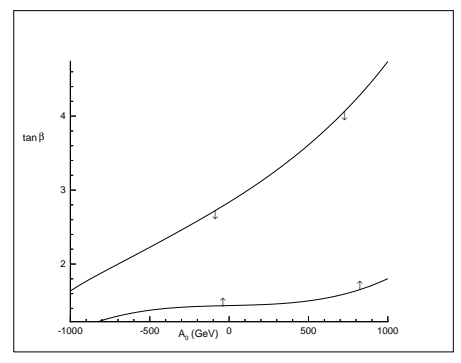

Fig. 4.b

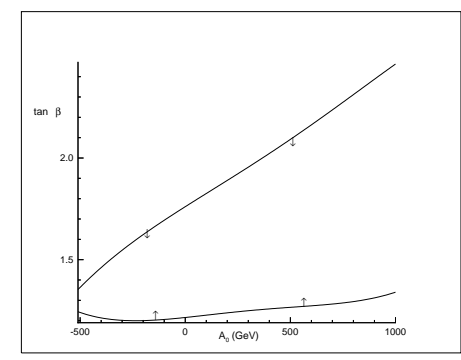

Fig. 4.c

Figure 4: a: The allowed region for $\tan \beta$ if we vary $\left|M_{1 / 2}\right|$ between 0 and $800 \mathrm{GeV} \cdot m_{0}=0 \mathrm{GeV}$ and $\lambda_{t}=0.8$ is fixed. $\mathrm{b}$ and $\mathrm{c}$ are the same as a with $m_{0}=500,1000 \mathrm{GeV}$.

space (which is now reduced to $m_{0}, M_{1 / 2}, A_{0}, \lambda_{t}$ ). These constraints displayed in Figs. 3 and 4 are the main results of our analysis. If the $\mu$-term is generated by the Higgs as PGB mechanism then the MSSM parameters must be inside the boundaries given in Figs. 3 and 4 or inside the tiny overlapping regions of Fig. 2.

Thus if one ultimately measures these parameters in colliders one can check whether they are indeed in the allowed region or not. If the MSSM parameters are all measured one can also check whether the experimental value of $\tan \beta$ does satisfy eq. 3.12 or not, thereby testing the assumptions on the GUT-scale Higgs sector.

\section{$5 \quad$ Implications for realistic models}

We finally comment on the validity of our analysis for models implementing the Higgs as PGB scheme. Although the Higgs as pseudo-Goldstone boson idea is perhaps the most natural solution to the doublet-triplet splitting problem in the context of SUSY GUT's, it is difficult to build realistic models that implement this idea in a natural way (see ref. [9]) without additional light charged particles which disrupt unification.

The only known realistic model is based on the $\mathrm{SU}(6)$ gauge group [ [ , 6, 7, 8, 9] and has an accidental $\mathrm{SU}(6) \otimes \mathrm{SU}(6)$ global symmetry of the Higgs sector. This symmetry is achieved by requiring that two sectors of the Higgs fields are are not mixed among each other in the superpotential. The models of refs. [4, 6, 7, 8, 9] use the $\mathrm{SU}(6)$ adjoint $\Sigma$ and a pair of $\mathrm{SU}(6)$ vectors $H, \bar{H}$ for the Higgs sector. Then the superpotential has the form

$$
W(\Sigma, H, \bar{H})=W_{1}(\Sigma)+W_{2}(H, \bar{H})
$$


up to dimension seven in the superpotential of the Higgs fields. This stringent requirement is necessary so that nonrenormalizable operators breaking the accidental global symmetry do not give too large a mass to the Higgs doublets. In ref. [9] several suggestions for a superpotential implementing this idea have been presented. From the point of view of the $\mu$-term we can divide them into two categories, according to whether a symmetry breaking term (that is a term that couples the $\Sigma$ and $H, \bar{H}$ fields) containing seven Higgs sector fields is or is not allowed. 2 A dimension seven operator would give an additional contribution to the $\mu$-term spoiling eq. 3.15 without destroying the solution to the doublet-triplet splitting problem.

In model 2 of ref. [9] such a term is allowed by all symmetries of the Lagrangian and thus may yield a contribution to the $\mu$-term of order $100-1000 \mathrm{GeV}$. Since the coefficient of this operator is a completely free parameter of the theory the constraint of eq. 3.15 does not hold and our analysis may not be applied to this theory. Such a theory cannot be tested by the constraints described in this paper.

However, if no dimension seven mixing terms are allowed in the superpotential then there can be no significant extra contribution to the $\mu$-term. This is the case in the simplest model, namely model 1 of ref. [9] and also in model 3 of the same reference.

The superpotential of model 1 of ref. [9] is given by

$$
\frac{1}{2} M \operatorname{Tr} \Sigma^{2}+\frac{1}{3} \lambda \operatorname{Tr} \Sigma^{3}+\frac{\alpha}{M_{P l}^{2 n-3}}(\bar{H} H)^{n},
$$

where $\mathrm{n}=4,5,6$. After the inclusion of the soft breaking terms one gets $\left.\langle H\rangle \sim 10^{17} \mathrm{GeV}\right\rangle$ $M_{G U T}$, and at this scale $\mathrm{SU}(6)$ is broken to $\mathrm{SU}(5)$. If one neglects the small admixture of $H, \bar{H}$ fields in the Higgs doublets then at the $\mathrm{SU}(5)$ scale $\langle H\rangle$ we have an $\mathrm{SU}(5)$ gauge theory with an "accidental" global SU(6) symmetry of the Higgs sector, since the theory originates from an SU(6) gauge theory. Thus at the $\langle H\rangle$ scale we get as an effective theory exactly the model of Section 2 since the SU(5) nonsinglet fields of $H$ are eaten by the heavy gauge bosons. This means that the threshold corrections to eq. 2.12 arising from the fact that the constraint is not generated at the GUT scale but at a somewhat higher scale can be estimated to be of the order

$$
\frac{\lambda_{t}^{2}}{16 \pi^{2}} \log \left(\frac{\langle H\rangle}{M_{G U T}}\right) \sim 0.01,
$$

due to the running between the $\langle H\rangle$ and GUT scales. Thus the corrections in this model to eq. 3.12 are expected to be a few percent and the results of our analysis should not be modified significantly. We have checked that corrections in eq. 2.12 as large as 10 percent

\footnotetext{
${ }^{2}$ The models in ref. 9] were especially designed such that no symmetry breaking terms containing only six or less Higgs sector fields are allowed, since these would give the doublet Higgses a mass of order $M_{G U T}\left(\frac{M_{G U T}}{M_{P l}}\right)^{-3} \sim 10^{7} \mathrm{GeV}$ and thus spoil the solution to the doublet-triplet splitting problem.
} 
caused only a small shift in the constraint curves. Consequently there was still no overlap between the allowed parameter region and the region allowed by CCB for the $\tan \beta>0$ case. Therefore the constraints obtained in this analysis should be robust.

\section{Conclusions}

We have investigated the consequences of the assumption that the supersymmetric $\mu$-term is entirely due to the Higgs as PGB mechanism. In this case the number of independent parameters is reduced by one; thus one can determine $\tan \beta$ as a function of the other parameters.

We have derived the additional constraint and examined its consequences. We found that for some region of input parameters this equation can not be fulfilled, constraining the possible parameter space. Namely we have found that one has either large $\left|A_{0}\right|$ and small $M_{1 / 2}$ and $m_{0}$, which is disfavored by the potentially dangerous presence of CCB vacua, or we get an ( $m_{0}$ and $A_{0}$ dependent) lower bound on $\left|M_{1 / 2}\right|$. In addition since $\tan \beta$ is determined by the other parameters, the allowed range of $\tan \beta$ is also constrained. Further experimental consequences might be revealed in subsequent probes of the restricted parameter space.

These bounds are consequences of the assumptions about the Higgs sector of the underlying GUT theory. Thus if SUSY is discovered, the measurement of the low energy parameters should also serve as a probe of the GUT scale physics.

\section{Acknowledgements}

We are grateful to Diego Castaño for many helpful suggestions and to Marcela Carena, Alex Kusenko and Carlos Wagner for useful discussions.

\section{References}

[1] K. Inoue, A. Kakuto and H. Takano, Prog. Theor. Phys. 75 (1986), 664.

[2] A. Anselm and A. Johansen, Phys. Lett. B200 (1988), 331;

A. Anselm, Sov. Phys. JETP 67 (1988), 663.

[3] R. Barbieri, G. Dvali and A. Strumia, Nucl. Phys. B391 (1993), 487.

[4] Z. Berezhiani and G. Dvali, Sov. Phys. Lebedev Inst. Rep. 5 (1989), 55.

[5] T. Goto, K. Inoue, Y. Okada and T. Yanagida, Phys. Rev. D46 (1992), R4808.

[6] R. Barbieri, G. Dvali and M. Moretti, Phys. Lett. B312 (1993), 137. 
[7] R. Barbieri, G. Dvali, A. Strumia, Z. Berezhiani and L. Hall, Nucl. Phys. 432 (1994), 49.

[8] Z. Berezhiani, INFN-FE-14-94, hep-ph/9412372;

Z. Berezhiani, Phys. Lett. B355 (1995), 481.

[9] Z. Berezhiani, C. Csáki and L. Randall, Nucl. Phys. B444 (1995), 61.

[10] L. E. Ibañez and C. López, Nucl. Phys. B233 (1984), 511;

L. E. Ibañez, C. López and C. Muñoz, Nucl. Phys. B256 (1985), 218.

[11] D. J. Castaño, E. J. Piard and P. Ramond, Phys. Rev. D49 (1994), 4882.

[12] M. Carena and C. E. M. Wagner, Nucl. Phys. B452 (1995), 45.

[13] A. Nelson and L. Randall, Phys. Lett. B316 (1993), 516.

[14] L. Montanet et. al. (Particle Data Group), Phys. Rev. D50 (1994), 1173;

M. Drees and S. P. Martin, MADPH-95-879, UM-TH-95-02, hep-ph/9504324.

[15] J. M. Frere, D. R. T. Jones and S. Raby, Nucl. Phys. B222 (1983), 11.

H. Komatsu, Phys. Lett. B215 (1988), 323.

J. F. Gunion, H. E. Haber and M. Sher, Nucl. Phys. B306 (1988), 1.

[16] J. A. Casas, A. Lleyda and C. Muñoz, FTUAM 95/11, hep-ph/9507294. 